\title{
La no obligatoriedad de la vacunación y su potencial impacto en la epidemiología de coqueluche
} Non-mandatory immunization and its potential impact on pertussis
epidemiology

\author{
Dra. Paula E. Bergero ${ }^{a}$ Dr. Gabriel Fabricius ${ }^{a}$ y Dra. Daniela F. Hozbor ${ }^{b}$
}

\begin{abstract}
RESUMEN
En este trabajo, se analizan cuantitativamente las consecuencias a corto plazo que tendría sobre coqueluche la sanción del Proyecto de Ley de Consentimiento Informado en Materia de Vacunación presentado en Argentina, en 2017, el cual contempla la no obligatoriedad de la aplicación de las vacunas del Calendario Nacional a los menores de edad. Se utiliza un modelo matemático para la transmisión de pertusis, desarrollado previamente en nuestro grupo. Se considera que la sola presentación del proyecto provoca una disminución en las coberturas por generar desconfianza sobre los beneficios del programa de vacunación. Asumiendo $5 \%$ anual de reducción de las coberturas durante 4 años a partir de 2018, en el siguiente brote, los casos graves en menores del año se incrementarían en más del $100 \%$ respecto del último brote, y se estiman 101 fallecidos. Con una reducción del $10 \%$ anual por 4 años, el siguiente brote superaría al previo en más del $200 \%$, con 163 decesos.

Palabras clave: cobertura devacunación, consentimiento informado, coqueluche.
\end{abstract}

http:/ / dx.doi.org/10.5546/aap.2018.418

Texto completo en inglés:

http:/ / dx.doi.org/10.5546/ aap.2018.eng.418

Cómo citar: Bergero PE, Fabricius G, Hozbor DF. La no obligatoriedad de la vacunación y su potencial impacto en la epidemiología de coqueluche. Arch Argent Pediatr 2018;116(6):418-421.

a. Grupo de Modelado y Simulación de Transmisión de Enfermedades Infecciosas, Instituto de Investigaciones Fisicoquímicas Teóricas y Aplicadas, Facultad de Ciencias Exactas, Universidad Nacional de La Plata y Centro Científico Tecnológico (CCT) de La Plata, CONICET de La Plata, Argentina.

b. Laboratorio VacSal, Instituto de Biotecnología y Biología Molecular, Departamento de Ciencias Biológicas, Facultad de Ciencias Exactas, Universidad Nacional de La Plata y CCT de La Plata, CONICET de La Plata, Argentina.

Correspondencia: Dra. Paula E. Bergero: paula_b@inifta.unlp.edu.ar

Financiamiento: Este trabajo fue financiado por la Agencia Nacional de Promoción Científica y Tecnológica (ANCPyT) (PICT 2014-3617, PICT 2012-2719).

Conflicto de intereses: Ninguno que declarar.

Recibido: 22-12-2017

Aceptado: 4-6-2018

Publicado Primero en Internet: 16-10-2018

\section{INTRODUCCIÓN}

A pesar del rol fundamental de la vacunación en el control de enfermedades infecciosas, sigue siendo, ocasionalmente, objetada o discutida. En varios países -Reino Unido, Suecia, Japón, entre otros-, las crisis de confianza en los programas de inmunización causaron el descenso de coberturas y / o la interrupción de la vacunación, lo que implica incidencias de la enfermedad respiratoria denominada coqueluche (o tos convulsa) entre 10 y 100 veces mayores que las reportadas donde no se afectaron los programas. ${ }^{1}$ En general, estas crisis de confianza se deben a acciones de grupos antivacunas o a eventos supuestamente atribuibles a la vacunación (ESAVI). Existen recomendaciones respecto de su manejo ante la opinión pública. ${ }^{2}$

En Argentina, la vacunación es gratuita y obligatoria desde 1983, según establece la Ley 22909. Sin embargo, en junio de 2017, fue presentado el Proyecto de Ley de Consentimiento Informado en Materia de Vacunación, que contemplaba la no obligatoriedad de las vacunas del Calendario al habilitar que se posibilitara la libre decisión de aceptar o rechazar la vacunación por parte de quienes fueran pasibles de vacunación o responsables de la vacunación de un menor de edad. ${ }^{3}$ La comunidad médica y académica rápidamente rechazó el proyecto y alertó sobre su efecto catastrófico en la incidencia de las enfermedades inmunoprevenibles. ${ }^{4-6}$ En caso de sancionarse esta ley, el efecto inmediato sería la disminución de las coberturas vacunales, lo que ocasionaría riesgo de reintroducción de enfermedades ya eliminadas (como difteria) y de agravamiento de otras endémicas (como coqueluche).

Pertusis es una infección respiratoria aguda inmunoprevenible potencialmente mortal en infantes. Como la efectividad de las vacunas disponibles es insuficiente para eliminarla, aun con altas coberturas, la enfermedad presenta brotes periódicos (cada 3-5 años). Actualmente la enfermedad es un problema para la salud pública. La vacunación contra coqueluche está indicada, en 
Argentina, a los 2, 4 y 6 meses de vida (esquema primario, quíntuple DTP-Hib-HB -vacuna combinada contra la difteria, el tétanos, la tos convulsa, Haemophilus influenzae y la hepatitis Bo cuádruple DTP-Hib), con refuerzos a los 18 meses, al ingreso escolar (triple bacteriana DTP), en la adolescencia, a embarazadas y cuidadores de la salud (triple acelular dTpa). ${ }^{7}$ Según datos oficiales, el esquema primario ha alcanzado, en los últimos años, coberturas próximas al $95 \%$ recomendado por la Organización Mundial de la Salud, aunque existen poblaciones debajo del $80 \%$. El brote reportado en Argentina, en 2011, causó 76 muertes de niños de un año o menos. ${ }^{8}$ A partir de 2012, siguiendo las recomendaciones internacionales, se incorporó el refuerzo para las embarazadas buscando proteger al neonato hasta recibir la primera dosis. El siguiente brote de coqueluche, ocurrido entre 2015 y 2016, tuvo $43 \%$ menos de casos graves en menores de un año. Según el Ministerio de Salud, en 2011 (antes de la vacunación materna), los menores de 2 meses constituyeron el 59,2\% de los decesos, mientras que, en 2015, representaron el 22,2\%. La tasa de letalidad cayó de 2,6\% en 2011 a $0,6 \%$ en $2016 .{ }^{9}$
Nuestra hipótesis es que tanto la presentación del mencionado proyecto como su discusión en la sociedad (aún sin ser aprobado) implican una pérdida de confianza de la población en las vacunas y una consecuente baja de las coberturas.

\section{OBJETIVO}

Analizar, mediante un modelado matemático, los efectos potenciales de la reducción de la cobertura de la vacunación sobre coqueluche.

\section{MÉTODOS}

Las consecuencias de la potencial disminución de las coberturas vacunales sobre la incidencia de casos graves de pertussis hasta el año de vida se analizaron a través de un modelo matemático de transmisión de la enfermedad determinista y estructurado en edades, que es descrito en la Figura 1. Este modelo matemático, desarrollado entre el Laboratorio VacSal (Instituto de Biotecnología y Biología Molecular -IBBM-, La Plata) y el Grupo de Modelado y Simulación de la Transmisión de Enfermedades Infecciosas del Instituto de Investigaciones Fisicoquímicas Teóricas y Aplicadas (INIFTA), fue previamente
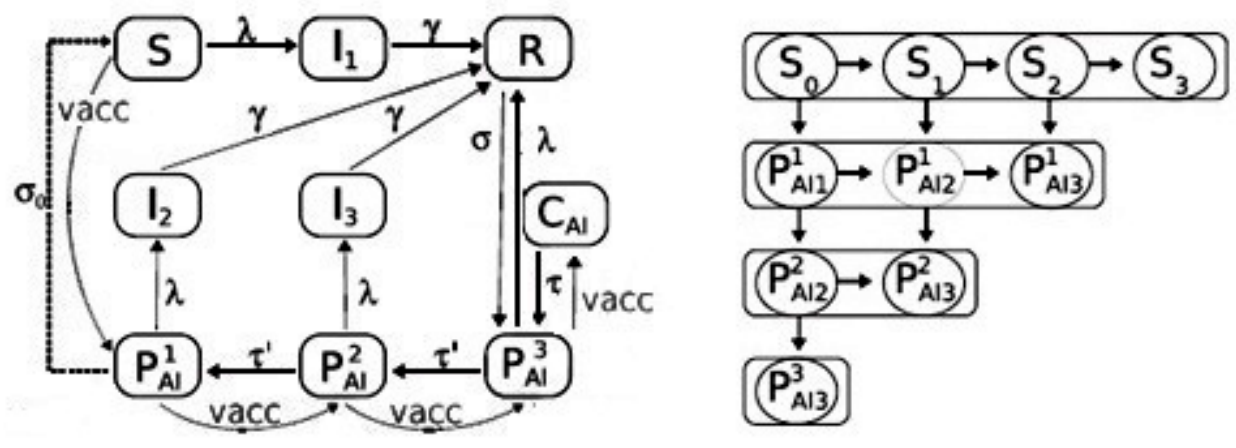

Izquierda: Los hijos de madres no vacunadas nacen en la clase susceptible S, donde permanecen a menos que se contagien y entren a la clase infecciosa $\mathrm{I}_{1}$ (que representa los casos graves) o sean vacunados al llegar a los 2 meses de edad y entren en el nivel más bajo de inmunidad parcial dado por la clase $\mathrm{P}_{\mathrm{AI1}}$ (partial acquired immunity, en inglés). Los hijos de madres vacunadas nacen, en cambio, ya en la clase de inmunidad parcial $\mathrm{P}_{\mathrm{Al} 1^{\prime}}$ donde permanecen por 2 meses, mientras les dura la protección de los anticuerpos. En ambos casos, al aplicarse sucesivas dosis efectivas de vacunas, los individuos irán adquiriendo inmunidad parcial (pasarán a las clases $\mathrm{P}_{\mathrm{Al} 2}$ y $\mathrm{P}_{\mathrm{Al} 3}$ ) hasta llegar, finalmente, a la inmunidad completa (clase $\mathrm{C}_{\mathrm{AI}}$; complete acquired immunity, en inglés). Los individuos en las clases parcialmente inmunes $\mathrm{P}_{\mathrm{AI} 1}$ y $\mathrm{P}_{\mathrm{AI} 2}$ también pueden contagiarse, pero desarrollan enfermedades progresivamente menos sintomáticas (y entran a las clases $\mathrm{I}_{2} \mathrm{e}_{3^{\prime}}$, respectivamente). Para los individuos en las clases infectivas, la infección desaparece luego de 21 días (es decir, salen de las clases $\mathrm{I}_{1}, \mathrm{I}_{2}$ e $\mathrm{I}_{3}$ a una tasa $\gamma=1 / 21$ [1/ día] y entran en la clase de recuperados $\mathrm{R}$ ). La inmunidad no dura toda la vida: se pierde con el tiempo a partir del año de vida a tasas $\tau=\tau^{\prime}=1 / 2, \sigma=1 / 11$ y $\sigma_{0}=1 / 100$ en unidades de $1 /$ año. ${ }^{10}$ Derecha: Detalle de la aplicación de vacunas. Las vacunas aplicadas no son $100 \%$ efectivas. Las flechas horizontales representan el efecto de la aplicación de dosis que resultan inefectivas (para esos individuos, aumenta el número de dosis recibidas, pero no la protección), mientras que las flechas verticales representan el efecto de la aplicación de dosis efectivas, y aumenta para los individuos que la reciben tanto el número de dosis como el nivel de inmunidad. Por simplicidad, se ha omitido, en la figura, la mortalidad correspondiente a cada compartimiento. Para detalles del modelo, véanse las referencias 10 a 12. 
presentado y validado, y ha sido usado para evaluar diferentes estrategias de control de coqueluche, como el refuerzo a adolescentes, el aumento de cobertura del esquema primario, la corrección de retrasos en la aplicación de dosis y la búsqueda de un calendario óptimo. ${ }^{10-13}$

En la situación epidemiológica referida, se modela y evalúa cuantitativamente el efecto de una caída gradual en las coberturas vacunales sobre la incidencia de coqueluche. La estimación de fallecidos se obtiene a partir de la incidencia calculada en el brote, considerando que son proporcionales a los casos graves y que, en 2011, hubo 76 de un año o menos.

El efecto de la vacunación materna sobre los lactantes fue modelado considerando que una fracción recibía pasivamente una inmunidad equivalente a una dosis de vacuna que perduraba hasta los 2 meses. ${ }^{12}$ La reducción de la fuente de contagio de los lactantes al tener madres inmunizadas no fue incluida aquí, ya que la evidencia en primates sugiere que la vacuna acelular anti coqueluche protegería respecto de la enfermedad, pero no evitaría la colonización ni la transmisión.

\section{Parámetros}

Los parámetros del modelo y la información epidemiológica utilizada para establecerlos se describen detalladamente en el trabajo de Pesco P. et al., ${ }^{11}$ Apéndice A, y corresponden al caso denominado CP1A-MDI (Contact Pattern1A - Medium Duration of Immunity).
La administración del esquema primario se parametrizó con datos del Hospital Elina de la Serna, de La Plata, que reflejaron el retraso con que la población se aplicaba cada dosis. ${ }^{13}$ La cobertura considerada para el esquema primario es $95 \%$; para los siguientes refuerzos, es $85 \%, 95 \%$ y $85 \%$, respectivamente, y $70 \%$ para la vacunación materna. Cada dosis tiene una efectividad de la vacuna $(E V)=0,9$, excepto la primera, considerada inferior debido a la inmadurez del sistema inmune del neonato, ${ }^{14} \mathrm{y}$ el refuerzo acelular a adolescentes (en ambos casos, $\mathrm{EV}=0,5$ ). Las fuerzas de infección $\lambda$ son parametrizadas a partir de casos reportados en la era prevacunal. ${ }^{10}$

\section{RESULTADOS}

La Figura 2 muestra la simulación de la incidencia de casos graves de coqueluche en Argentina en menores de un año. El estado estacionario del modelo fue perturbado para emular un brote similar al de 2011. La vacunación a embarazadas se inició en 2012. En el panel izquierdo, se supone que el proyecto de ley comenzará a ser tratado en 2018, y se iniciará, entonces, la hipotética disminución de coberturas de todas las dosis durante 4 años. Se consideran dos escenarios de descenso de coberturas: a) $5 \%$ anual hasta un total de $20 \%$; b) $10 \%$ anual hasta un total de $40 \%$. El escenario a) es compatible con el descenso reportado en Chile, ${ }^{15}$ cuyo movimiento antivacunas es relativamente poco activo, como en Argentina, mientras que el b) se aproxima al reportado en países donde los

Figura 2. Resultados del modelo matemático para la incidencia de casos graves de pertussis hasta el año de edad.
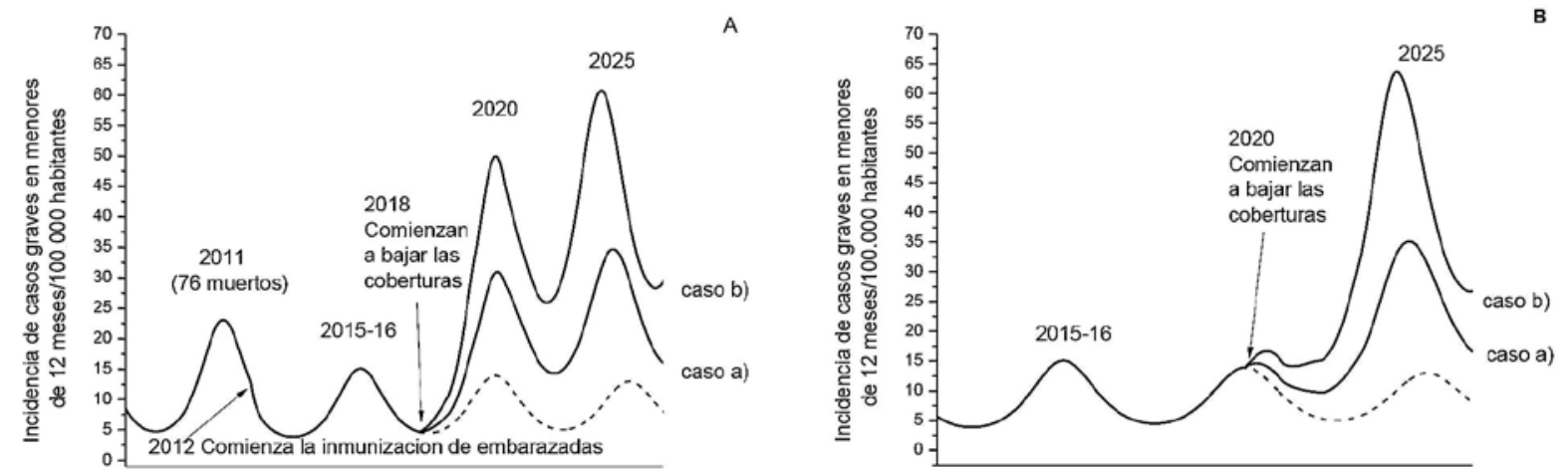

Panel A: Simulación de la epidemiología de la tos convulsa en menores de un año en Argentina y el efecto de una hipotética baja en todas las coberturas vacunales. Caso a): reducción de $5 \%$ anual en las coberturas; caso b): reducción de $10 \%$ anual en las coberturas. El descuido de coberturas se inicia a partir de 2018, es decir, en el mínimo de incidencia. Panel B: La disminución de coberturas comienza en 2020, es decir, en el máximo de incidencia (brote). La línea de puntos indica la evolución de la incidencia cuando no se registra la baja en las coberturas. 
programas fueron seriamente afectados. ${ }^{1}$

Nuestro modelo indica que, si se redujeran, a partir de 2018, las coberturas de todas las dosis, según el caso a), en el brote de 2020, los casos graves se incrementarían en más del $100 \%$ respecto del brote anterior (2015), con 101 decesos estimados (Figura 2, panel A). En el caso de una reducción mayor (caso b), el siguiente brote superaría al previo en más del $200 \%$ y los decesos se estimarían en 163.

Como cualquier intervención en un sistema dinámico genera un efecto diferente según en qué momento del ciclo ocurra, en la Figura 2, panel B, se explora el sistema con la baja de coberturas que se iniciaría en 2020. Los incrementos en el brote siguiente (2025) respecto del brote anterior serían de más del $100 \%$ con estimación de 115 fallecidos (caso a) y de más del $300 \%$ con estimación de 209 fallecidos (caso b).

\section{DISCUSIÓN}

Nuestro estudio sugiere que, considerando distintos escenarios posibles de coberturas y año de inicio del descenso de cobertura, y aun en el caso considerado como menos desfavorable (es decir, $5 \%$ anual de reducción de coberturas durante 4 años a partir de 2018), en el siguiente brote de tos convulsa, los casos graves en menores del año se incrementarían en más del $100 \%$ y aumentarían también los fallecidos.

La incerteza existente en los valores de ciertos parámetros, como los contagios entre diferentes grupos etarios, representa una limitación para este estudio. Las incidencias presentadas aquí podrían variar al calcularlas con otros valores de parámetros epidemiológicamente compatibles. En análisis de sensibilidad previos, se ha estudiado cómo varía la incidencia ante un cambio de cobertura bajo distintos conjuntos de parámetros y se concluye que las tendencias predichas son robustas. ${ }^{10,11}$

Nuestros resultados sostienen que alcanzar $-y$ mantener- altas coberturas en las dosis primarias y a embarazadas resulta la estrategia principal para evitar casos de pertusis en lactantes. ${ }^{10-13}$ Sin embargo, para mantener las coberturas altas y la confianza de la comunidad en el programa de inmunización, es necesario generar campañas que comuniquen los beneficios y alerten sobre las consecuencias de una vacunación incompleta o retrasada. En este sentido, creemos que este trabajo resulta un aporte, ya que se presentan resultados de un modelo matemático robusto y parametrizado localmente que permiten cuantificar el riesgo, respecto de coqueluche, que conlleva poner en peligro el programa de inmunización obligatoria.

\section{REFERENCIAS}

1. Gangarosa EJ, Galazka AM, Wolfe CR, et al. Impact of antivaccine movements on pertussis control: the untold story. Lancet 1998; 351(9099):356-61.

2. Organización Panamericana de la Salud. Vacunación Segura: Cómo enfrentar los eventos supuestamente atribuidos a la vacunación o inmunización. Washington DC, EE. UU.: OPS; 2002. [Acceso: 5 de junio de 2017]. Disponible en: http://www.who.int/immunization_ safety / publications / aefi/en/vacunacion_segura_S.pdf.

3. Urroz PM. Consentimiento informado en materia de vacunación. Proyecto de Ley. Buenos Aires; 2017. [Acceso: noviembre de 2017]. Disponible en: http://www4. hcdn.gob.ar/dependencias / dsecretaria / Periodo2017/ PDF2017 / TP2017/2467-D-2017.pdf.

4. Comisión Directiva de la Sociedad Argentina de Pediatría. Vacunarse es obligatorio y un derecho de todos. [Acceso: junio de 2017]. Disponible en: http://www.sap.org. ar/uploads / documentos / documentos_vacunarse-esobligatorio-y-un-derecho-de-todos-113.pdf.

5. Sociedad Argentina de Inmunología. Comunicado Oficial. 2017. [Acceso: junio de 2017]. Disponible en: http:// inmunologia.org.ar/comunicado-oficial/.

6. Grieco G, Zamponi A. Movimientos antivacunas: una moda peligrosa 2017. [Acceso: julio de 2017]. Disponible en: http: / / www.unsam.edu.ar/tss / movimientosantivacunas-una-moda-peligrosa/.

7. Ministerio de Salud de la Nación. Calendario Nacional de Vacunación de la República Argentina. 2017. [Acceso: 5 de junio de 2017]. Disponible en: https://www.argentina. gob.ar/sites / default / files / salud_vacunas_calendario_ nacional_vacunacion_2017.pdf.

8. Romanin V, Agustinho V, Califano G, et al. Situación epidemiológica de coqueluche y estrategias para su control. Argentina, 2002-2011. Arch Argent Pediatr 2014;112(5):413-20.

9. Ministerio de Salud. Boletín Integrado de Vigilancia. 2017;349. [Acceso: 5 de junio de 2017]. Disponible en: https: / / www.argentina.gob.ar / sites / default / files / boletin_integrado_vigilancia_n349-se8.pdf.

10. Fabricius G, Bergero PE, Ormazabal ME, et al. Modelling pertussis transmission to evaluate the effectiveness of an adolescent booster in Argentina. Epidemiol Infect 2013; 141(4):718-34.

11. Pesco P, Bergero P, Fabricius G, Hozbor D. Mathematical modeling of delayed pertussis vaccination in infants. Vaccine 2015;33(41):5475-80.

12. Bergero PE, Fabricius G, Hozbor DF, et al. Potential Impact of Changes in the Schedule for Primary Diphtheria-Tetanus Toxoids-Pertussis Immunization as Control Strategy for Pertussis. Pediatr Infect Dis J 2018; 37(2):e36-42.

13. Pesco P, Bergero P, Fabricius G, Hozbor D. Evaluación delas estrategias de vacunación contra la tos convulsa mediante un modelo matemático de transmisión de la enfermedad. Arch Argent Pediatr 2013; 111(5):377-83.

14. Pertussis Working Group. Report from the SAGE Working Group on Pertussis Vaccines 2014. [Acceso: 5 de junio de 2017]. Disponible en: http:/ / www.who.int/ immunization/sage/meetings/2015/april/1_Pertussis_ report_final.pdf.

15. Herrera Cabello J. Cae cobertura de vacunas obligatorias a nivel nacional en 2016. La Tercera, Chile. (Grupo Copesa). 23 de enero de 2017. 